\title{
SPONTANEOUS MALE CROSSING-OVER OF FREQUENT OCCURRENCE IN DROSOPHILA ANANASSAE FROM SOUTHEAST ASIAN POPULATIONS
}

\author{
DAIGORO MORIWAKI AND YOSHIKO N. TOBARI \\ National Institute of Genetics, Misima, Sizuoka-ken 411 \\ and \\ Department of Biology, Tokyo Metropolitan University \\ Setagaya-ku, Tokyo 158
}

Received January 13, 1973

Spontaneous occurrence of male crossing-over was first observed by Moriwaki (1937, 1938, 1940) and Kikkawa (1937) in D. ananassae independently. They concluded that the events were under genetic control. Lately, as to this phenomenon several authors published data which demonstrated that the male crossing-over in this species was controlled by several genes or polygenes and mainly occurred in meiotic stage (Mukherjee 1961, Kale 1969, Moriwaki et al. 1970, Hinton 1970). As stated above, in $D$. ananassae, in contrast with other species of Drosophila, male crossing-over has rather often been observed. However, little is known about proportion of chromosomes responsible for spontaneous male crossing-over in natural populations.

In the present experiment frequencies of chromosomes which produced spontaneous male crossing-over in $D$. ananassae of Southeast Asian populations were examined.

\section{MATERIALS AND METHODS}

From May to September in 1971, a research group presented by Dr. K. Wakahama of Shimane University explored many localities over Southeast, Mideast Asia and Africa, collecting Drosophila species. By the member's aid, several isofemale lines of D. ananassae from each locality were made and shipped by air mail to Tokyo. Thanks are due to their help.

The isofemale lines of $D$. ananassae used in this study were established from single inseminated females collected in nature from six localities as follows: Taiwan (A), Kota Kinabaru, Borneo (B), Kuala Lumpur, Malaysia (C), Chian Mai, Thailand (D), Calcutta, India (E) and Colombo, Ceylon (L) (Fig. 1). Five isofemale lines from each locality, but three in $\mathrm{E}$, were examined in male crossing-over frequencies within several generations after collection. Five males from each line were singly mated with double recessive females, $b$ se (black sepia) on the second chromosome. One day old single $\mathrm{F}_{1}$ male $(b \mathrm{se} /++)$ recovered from each cross was backcrossed with $b$ se virgin females.

1) This work was supported by a grant from the Scientific Research Fund of the Ministry of Education, Japan, No. 50801. 


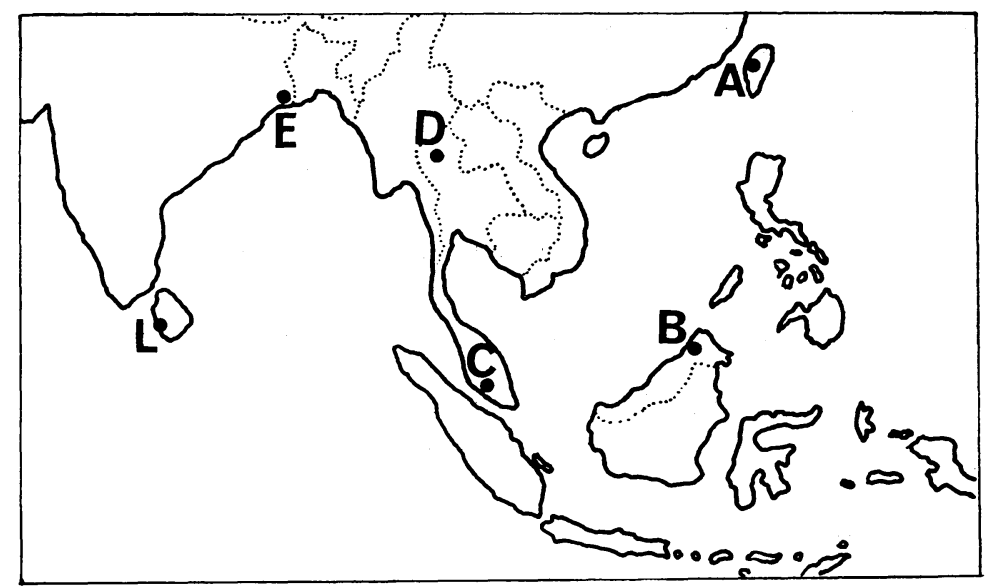

Fig. 1. Localities from which $D$. ananassae were examined for male crossing-over.

After a three or four day egg laying period, parents were transferred to new vials successively twice. Thus three 3-4 day broods were provided. Progenies recovered from each brood of each family were scored. The experiments were performed in the constant temperature room at $25^{\circ} \mathrm{C}$.

\section{RESULTS}

The data of three broods were pooled to calculate the recombination values in males between $b$ and $s e$. The results were summarized in Table 1. As shown in the table, the male crossing-over occurred in 133 chromosomes (95\%) of 140 tested. In the four populations, $\mathrm{A}, \mathrm{B}, \mathrm{C}$ and $\mathrm{E}$, among six, all chromosomes tested were observed to show male crossing-over. In most of the lines of six localities great variations from male to male were observed in recombination value. These values ranged from zero to about $20 \%$. Significant differences in recombination values were detected among lines but not among localities by the analysis of variance.

The mean recombination value of all 140 chromosomes from six localities amounted to about $4.0 \%$, while in females it was $48.8 \%$. In other words, the male recombination value between $b$ and $s e$ was about $8 \%$ of the female one.

The equality of complementary crossover classes in each family was examined following Fisher's method used by Hinton and Whittinghill (1950). In the Appendix Table, probabilities of obtaining the observed distributions of crossover classes in each family and respective $-\log _{\theta}$ value are presented. The $-\log _{e}$ value is equal to $\chi^{2} / 2$ associated with 2 degrees of freedom and additive in character. Only two out of 125 families studied are significantly deviated from equality. Therefore it will be concluded that the equality of complementary crossover classes is demonstrated in almost all families with only two exceptions.

Correlation between recombination frequency and $\mathrm{k}$ value was computed, where $\mathrm{k}=$ (wild type $+1 / 2$ recombinant)/total progeny. The recombination values were used 
Table 1. Recombination values between $b$ and $s e$ in males, calculated for each line of six localities

\begin{tabular}{|c|c|c|c|c|c|c|c|c|c|c|}
\hline \multirow{3}{*}{$\begin{array}{l}\text { Locality } \\
\text { TAIWAN }\end{array}$} & \multirow{2}{*}{$\begin{array}{l}\text { Line } \\
\text { A3 } \\
\text { A5 } \\
\text { A31 } \\
\text { A36 } \\
\text { A75 }\end{array}$} & \multirow{3}{*}{$\begin{array}{c}\begin{array}{c}\text { No. of } \\
\text { chromosome } \\
\text { tested }\end{array} \\
5 \\
5 \\
5 \\
5 \\
5 \\
5 \\
25\end{array}$} & \multirow{3}{*}{$\begin{array}{c}\begin{array}{l}\text { No. of } \\
\text { chr. with } \\
\text { male c.o. } \\
5 \\
5 \\
5 \\
5 \\
5 \\
25\end{array}\end{array}$} & \multicolumn{2}{|c|}{$\begin{array}{c}\text { Proge } \\
\text { Non-recom- } \\
\text { binant } \\
b \text { se + }\end{array}$} & \multicolumn{2}{|c|}{$\begin{array}{l}\text { ny } \\
\text { Recom- } \\
\text { binant } \\
b \text { se }\end{array}$} & $\mathrm{N}$ & $\begin{array}{l}\text { Mean } \\
\text { R.V. } \\
(\%)\end{array}$ & $\begin{array}{c}\text { Range of } \\
\text { R.V. } \\
(\%)\end{array}$ \\
\hline & & & & $\begin{array}{r}716 \\
784 \\
930 \\
1115 \\
931\end{array}$ & $\begin{array}{r}689 \\
944 \\
1176 \\
1334 \\
1135\end{array}$ & $\begin{array}{l}35 \\
31 \\
36 \\
37 \\
49\end{array}$ & $\begin{array}{l}26 \\
26 \\
26 \\
38 \\
46\end{array}$ & $\begin{array}{l}1466 \\
1785 \\
2168 \\
2524 \\
2161\end{array}$ & $\begin{array}{l}3.90 \\
3.12 \\
2.67 \\
2.98 \\
4.57\end{array}$ & $\begin{array}{l}2.29-5.62 \\
2.05-4.25 \\
0.17-6.06 \\
1.20-4.39 \\
1.74-6.74\end{array}$ \\
\hline & Total & & & 4476 & 5278 & 188 & 162 & 10104 & 3.45 & \\
\hline \multirow[t]{2}{*}{ BORNEO } & $\begin{array}{l}\text { B13 } \\
\text { B15 } \\
\text { B18 } \\
\text { B42 } \\
\text { B45 }\end{array}$ & $\begin{array}{l}5 \\
5 \\
5 \\
5 \\
5\end{array}$ & $\begin{array}{l}5 \\
5 \\
5 \\
5 \\
5\end{array}$ & $\begin{array}{l}467 \\
407 \\
408 \\
532 \\
558\end{array}$ & $\begin{array}{l}538 \\
436 \\
574 \\
547 \\
596\end{array}$ & $\begin{array}{l}15 \\
17 \\
40 \\
16 \\
14\end{array}$ & $\begin{array}{r}20 \\
6 \\
35 \\
16 \\
14\end{array}$ & $\begin{array}{r}1040 \\
866 \\
1057 \\
1111 \\
1182\end{array}$ & $\begin{array}{l}3.42 \\
2.67 \\
7.61 \\
2.35 \\
2.42\end{array}$ & $\begin{array}{l}1.59-5.65 \\
1.79-3.65 \\
4.87-11.97 \\
0.55-5.07 \\
0.61-4.39\end{array}$ \\
\hline & Total & 25 & 25 & 2372 & 2691 & 102 & 91 & 5256 & 3.69 & \\
\hline \multirow[t]{2}{*}{ MALAYSIA } & $\begin{array}{l}\text { C104 } \\
\text { C105 } \\
\text { C106 } \\
\text { C107 } \\
\text { C123 }\end{array}$ & $\begin{array}{l}5 \\
5 \\
5 \\
5 \\
5\end{array}$ & $\begin{array}{l}5 \\
5 \\
5 \\
5 \\
5\end{array}$ & $\begin{array}{r}504 \\
325 \\
700 \\
1064 \\
989\end{array}$ & $\begin{array}{r}560 \\
404 \\
739 \\
1198 \\
1091\end{array}$ & $\begin{array}{l}45 \\
17 \\
38 \\
47 \\
43\end{array}$ & $\begin{array}{l}33 \\
18 \\
39 \\
31 \\
44\end{array}$ & $\begin{array}{r}1142 \\
764 \\
1516 \\
2340 \\
2167\end{array}$ & $\begin{array}{l}6.54 \\
4.48 \\
5.44 \\
3.34 \\
4.28\end{array}$ & $\begin{array}{l}2.91-16.07 \\
2.17-7.38 \\
3.49-9.52 \\
2.91-4.77 \\
0.79-8.67\end{array}$ \\
\hline & Total & 25 & 25 & 3582 & 3992 & 190 & 165 & 7929 & 4.82 & \\
\hline \multirow[t]{2}{*}{ THAILAND } & $\begin{array}{l}\text { D20 } \\
\text { D21 } \\
\text { D23 } \\
\text { D25 } \\
\text { D28 }\end{array}$ & $\begin{array}{l}5 \\
5 \\
5 \\
5 \\
5\end{array}$ & $\begin{array}{l}5 \\
5 \\
5 \\
4 \\
4\end{array}$ & $\begin{array}{l}517 \\
410 \\
567 \\
347 \\
417\end{array}$ & $\begin{array}{l}678 \\
552 \\
625 \\
399 \\
475\end{array}$ & $\begin{array}{r}31 \\
33 \\
18 \\
9 \\
24\end{array}$ & $\begin{array}{l}36 \\
33 \\
17 \\
10 \\
23\end{array}$ & $\begin{array}{r}1262 \\
1028 \\
1227 \\
765 \\
939\end{array}$ & $\begin{array}{l}5.14 \\
6.39 \\
2.49 \\
2.48 \\
5.62\end{array}$ & $\begin{array}{ll}2.58-9.00 & 9.00-10.43 \\
0.62-4.01 \\
0 & -5.84 \\
0 & -13.91\end{array}$ \\
\hline & Total & 25 & 23 & 2258 & 2729 & 115 & 119 & 5221 & 4.42 & \\
\hline \multirow[t]{2}{*}{ CALCUTTA } & $\begin{array}{l}\text { E22 } \\
\text { E23 } \\
\text { E24 }\end{array}$ & $\begin{array}{l}5 \\
5 \\
5\end{array}$ & $\begin{array}{l}5 \\
5 \\
5\end{array}$ & $\begin{array}{l}408 \\
359 \\
495\end{array}$ & $\begin{array}{l}495 \\
384 \\
534\end{array}$ & $\begin{array}{r}9 \\
15 \\
25\end{array}$ & $\begin{array}{r}7 \\
13 \\
21\end{array}$ & $\begin{array}{r}919 \\
771 \\
1075\end{array}$ & $\begin{array}{l}1.84 \\
3.99 \\
4.52\end{array}$ & $\begin{array}{l}0.97-2.83 \\
0.74-10.66 \\
1.75-6.38\end{array}$ \\
\hline & Total & 15 & 15 & 1262 & 1413 & 49 & 41 & 2765 & 3.45 & \\
\hline \multirow[t]{2}{*}{ COLOMBO } & $\begin{array}{l}\text { L2 } \\
\text { L5 } \\
\text { L7 } \\
\text { L8 } \\
\text { L10 }\end{array}$ & $\begin{array}{l}5 \\
5 \\
5 \\
5 \\
5\end{array}$ & $\begin{array}{l}5 \\
5 \\
2 \\
5 \\
3\end{array}$ & $\begin{array}{l}321 \\
412 \\
350 \\
322 \\
438\end{array}$ & $\begin{array}{l}371 \\
513 \\
354 \\
447 \\
500\end{array}$ & $\begin{array}{r}10 \\
12 \\
9 \\
51 \\
10\end{array}$ & $\begin{array}{r}14 \\
8 \\
8 \\
33 \\
4\end{array}$ & $\begin{array}{l}716 \\
945 \\
721 \\
853 \\
952\end{array}$ & $\begin{array}{r}3.29 \\
1.82 \\
1.82 \\
11.28 \\
1.44\end{array}$ & $\begin{array}{l}1.34-6.33 \\
0.47-3.57 \\
0-7.33 \\
1.95-20.47 \\
0 \quad-3.80\end{array}$ \\
\hline & Total & 25 & 20 & 1843 & 2185 & 92 & 67 & 4187 & 3.93 & \\
\hline
\end{tabular}

after arcsine transformation. Slightly significant positive correlation was detected $(\mathrm{r}=$ $0.170, \mathrm{t}=2.034,0.05>\mathrm{p}>0.025)$. In Fig. 2, recombination value of each family after arcsine transformation was plotted at the corresponding $\mathrm{k}$ value.

\section{DISCUSSION}

The equality of complementary crossover classes was demonstrated, suggesting the meiotic origin of the event. Wide individual variation and significant differences of recombination values among lines may attribute to polygenic systems (Moriwaki et al. 1970). 


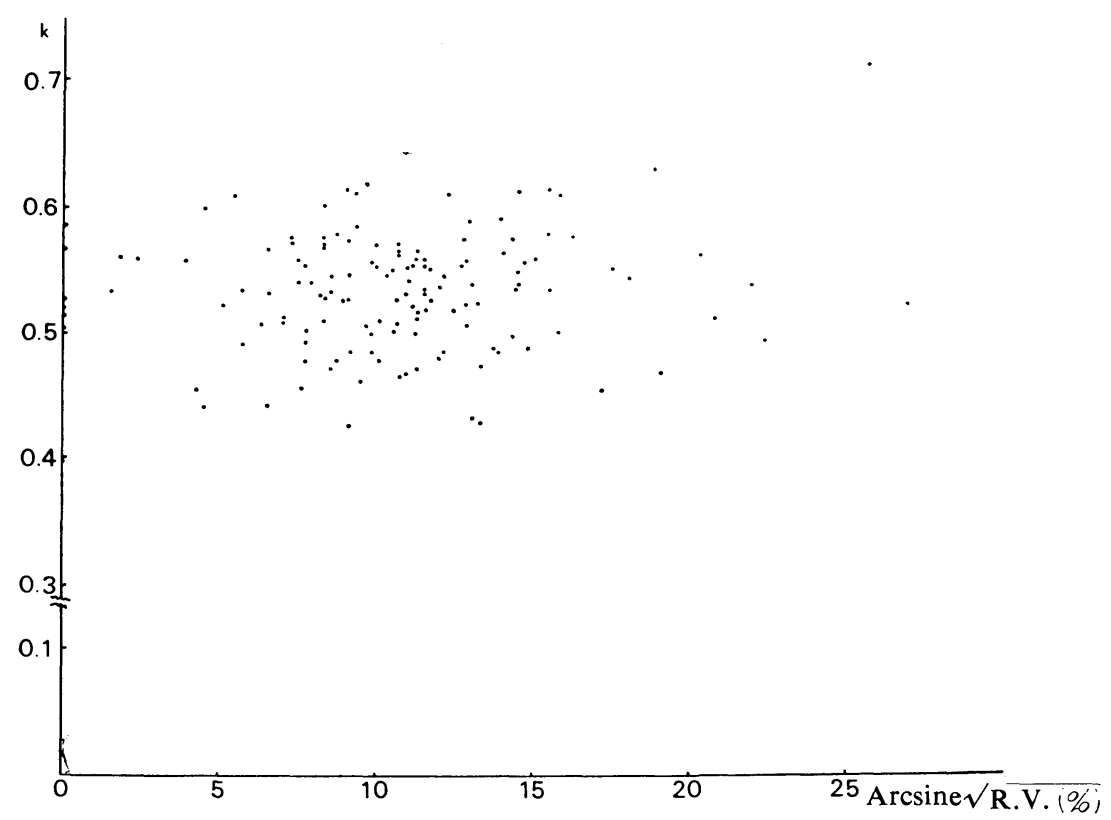

Fig. 2. Distribution of recombination values plotted against $\mathrm{k}$ values of males in D. ananassae.

Hiraizumi (1971) reported highly significant negative regression of recombination frequency on the $\mathrm{k}$ value in $D$. melanogaster. In contrast with that, the present experiment represented positive correlation between recombination and $\mathrm{k}$ values. However, meanings of the property are not explained at this moment.

In the male of Drosophila there is normally no crossing-over. In the female inversion heterozygosity leads to extensive crossing-over reduction. Consequently they prevent recombination in a balanced gene complex. One way to maintain balance between immediate fitness and future flexibility of Drosophila in natural populations is the storage and release of potential genetic variability which are controlled by the amount of inversion heterozygosity and extreme difference in recombination between sexes (Bodmer and Parsons 1962).

In the present study amaizingly large proportion of chromosomes in Southeast Asian populations was observed to produce spontaneous male crossing-over. In laboratory stocks originated from various localities of $D$. ananassae about $65 \%$ of chromosomes were found to have male crossing-over (Moriwaki et al. 1970). Therefore, in D. ananassae populations male recombination may not a little contribute to the release of the populations. More extensive survey over the range of species distribution with regards to male crossing-over and distribution of inversions may provide a mode of compromise between immediate fitness and future flexibility of the species. 


\section{SUMMARY}

Drosophila ananassae from six geographical localities in Southeast Asia were examined in the recombination frequencies in the male. The investigation reveals that male crossing-over may be very common. At least in the second chromosome from these populations, almost all males can be expected to carry some genetic elements to cause male recombination, although in general the recombination value in the male was not always so high as that in the female.

The equality of complementary crossover classes in each family was observed suggesting the meiotic origin of the event.

Positive correlation between recombination and $\mathrm{k}$ values was obtained.

\section{ACKNOWLEDGMENTS}

The authors wish to express their thanks to Miss S. Serizawa for her technical assistance.

\section{LITERATURE CITED}

Bodmer, W. F., and P. A. Parsons, 1962 Linkage and recombination in evolution. Advances in Genetics 11: 1-100.

Hinton, C. W., 1970 Identification of two loci controlling crossing over in males of Drosophila ananassae. Genetics 66: 663-676.

Hinton, C. W., and M. Whittinghill, 1950 The distribution of X-ray induced crossovers from Curly inversion heterozygotes of Drosophila melanogaster females. Proc. Natl. Acad. Sci. 36: $552-558$.

Hiraizumi, Y., 1971 Spontaneous recombination in Drosophila melanogaster males. Proc. Natl. Acd. Sci. 68: 268-270.

Kale, P. G., 1969 The meiotic origin of spontaneous crossovers in Drosophila ananassae. Genetics 62: 123-133.

Kikkawa, H., 1937 Spontaneous crossing-over in the male of Drosophila ananassae. Zool. Mag. 49: $159-160$.

Moriwaki, D., 1937 A high ratio of crossing over in Drosophila ananassae. Ztschr. indukt. Abstammungs-u. Vererb. 74: 17-23.

Moriwaki, D., 1938 Enhanced crossing over over in the second chromosome of Drosophila ananassae. (in Japanese). Japan. J. Genetics 14: 283-284.

Moriwaki, D., 1940 Enhanced crossing over in the second chromosome of Drosophila ananassae. Japan. J. Genetics 16: 37-48.

Moriwaki, D., Y. N. Tobari, and Y. Oguma, 1970 Spontaneous crossing-over in the male of Drosophila ananassae. Japan. J. Genetics 45: 411-420.

Mukherjee, A. S., 1961 Effect of selection on crossing over in the males of Drosophila ananassae. Am. Naturalist 95: 57-59. 
Appendix Table. Familial distribution of recombinants in offspring from $b \mathrm{se} /+$ males and Chi square analysis for testing the equality of complementary crossovers within families

\begin{tabular}{|c|c|c|c|c|c|c|c|c|c|}
\hline & $b$ & se & $\mathrm{p}$ & $-\log _{e}$ & & $b$ & se & $\mathrm{p}$ & $-\log _{e}$ \\
\hline A $\begin{array}{rr}3-1 \\
\\
\\
\\
& 2 \\
& 3 \\
& 4 \\
& 5\end{array}$ & $\begin{array}{r}10 \\
8 \\
6 \\
9 \\
2\end{array}$ & $\begin{array}{r}9 \\
4 \\
1 \\
10 \\
2\end{array}$ & $\begin{array}{l}0.500 \\
0.194 \\
0.063 \\
0.500 \\
0.500\end{array}$ & $\begin{array}{l}0.693 \\
1.640 \\
2.765 \\
0.693 \\
0.693\end{array}$ & $\begin{array}{r}\text { B13-1 } \\
2 \\
3 \\
4 \\
5\end{array}$ & $\begin{array}{l}1 \\
1 \\
5 \\
3 \\
5\end{array}$ & $\begin{array}{l}3 \\
3 \\
1 \\
4 \\
9\end{array}$ & $\begin{array}{l}0.313 \\
0.313 \\
0.109 \\
0.500 \\
0.212\end{array}$ & $\begin{array}{l}1.161 \\
1.161 \\
2.216 \\
0.693 \\
1.551\end{array}$ \\
\hline $\begin{array}{rr}\text { A } 5-1 \\
\\
\\
\\
\\
\\
& 2 \\
& 3 \\
& 4\end{array}$ & $\begin{array}{r}4 \\
7 \\
7 \\
10 \\
3\end{array}$ & $\begin{array}{l}5 \\
6 \\
7 \\
7 \\
1\end{array}$ & $\begin{array}{l}0.500 \\
0.500 \\
0.500 \\
0.315 \\
0.313\end{array}$ & $\begin{array}{l}0.693 \\
0.693 \\
0.693 \\
1.155 \\
1.161\end{array}$ & $\begin{array}{r}\text { B15-1 } \\
2 \\
3 \\
4 \\
5\end{array}$ & $\begin{array}{l}3 \\
3 \\
3 \\
3 \\
5\end{array}$ & $\begin{array}{l}2 \\
1 \\
0 \\
1 \\
2\end{array}$ & $\begin{array}{l}0.500 \\
0.313 \\
0.125 \\
0.313 \\
0.227\end{array}$ & $\begin{array}{l}0.693 \\
1.161 \\
2.080 \\
1.161 \\
1.483\end{array}$ \\
\hline $\begin{array}{r}\text { A } 31-1 \\
2 \\
3 \\
4 \\
5\end{array}$ & $\begin{array}{r}6 \\
1 \\
14 \\
14 \\
1\end{array}$ & $\begin{array}{r}6 \\
0 \\
14 \\
6 \\
0\end{array}$ & $\begin{array}{l}0.500 \\
\\
0.500 \\
0.058\end{array}$ & $\begin{array}{l}0.693 \\
2.847\end{array}$ & $\begin{array}{r}\text { B18-1 } \\
2 \\
3 \\
4 \\
5\end{array}$ & $\begin{array}{r}9 \\
4 \\
12 \\
4 \\
11\end{array}$ & $\begin{array}{r}8 \\
5 \\
12 \\
6 \\
4\end{array}$ & $\begin{array}{l}0.500 \\
0.500 \\
0.500 \\
0.377 \\
0.059\end{array}$ & $\begin{array}{l}0.693 \\
0.693 \\
0.693 \\
0.975 \\
2.830\end{array}$ \\
\hline $\begin{array}{r}\text { A } 36-1 \\
2 \\
3 \\
4 \\
5\end{array}$ & $\begin{array}{r}9 \\
7 \\
3 \\
7 \\
11\end{array}$ & $\begin{array}{r}16 \\
4 \\
2 \\
7 \\
9\end{array}$ & $\begin{array}{l}0.115 \\
0.274 \\
0.500 \\
0.500 \\
0.412\end{array}$ & $\begin{array}{l}2.163 \\
1.295 \\
0.693 \\
0.693 \\
0.887\end{array}$ & $\begin{array}{r}\text { B } 42-1 \\
2 \\
3 \\
4 \\
5\end{array}$ & $\begin{array}{l}5 \\
1 \\
1 \\
8 \\
1\end{array}$ & $\begin{array}{r}10 \\
0 \\
1 \\
5 \\
0\end{array}$ & $\begin{array}{l}0.500 \\
0.291\end{array}$ & $\begin{array}{l}1.890 \\
0.693 \\
1.234\end{array}$ \\
\hline $\begin{array}{r}\text { A } 70-1 \\
2 \\
3 \\
4 \\
5\end{array}$ & $\begin{array}{r}9 \\
8 \\
3 \\
14 \\
15\end{array}$ & $\begin{array}{r}7 \\
6 \\
3 \\
13 \\
17\end{array}$ & $\begin{array}{l}0.402 \\
0.395 \\
0.500 \\
0.500 \\
0.430\end{array}$ & $\begin{array}{l}0.911 \\
0.929 \\
0.693 \\
0.693 \\
0.844\end{array}$ & $\begin{array}{r}\text { B } 45-1 \\
2 \\
3 \\
4 \\
5\end{array}$ & $\begin{array}{l}0 \\
5 \\
3 \\
2 \\
4\end{array}$ & $\begin{array}{l}1 \\
1 \\
7 \\
1 \\
4\end{array}$ & $\begin{array}{l}0.109 \\
0.172 \\
0.500 \\
0.500\end{array}$ & $\begin{array}{l}2.216 \\
1.760 \\
0.693 \\
0.693\end{array}$ \\
\hline $\begin{array}{r}\mathrm{C} 104-1 \\
2 \\
3 \\
4 \\
5\end{array}$ & $\begin{array}{r}24 \\
5 \\
7 \\
5 \\
4\end{array}$ & $\begin{array}{r}21 \\
3 \\
1 \\
3 \\
5\end{array}$ & $\begin{array}{l}0.383 \\
0.363 \\
0.035 \\
0.363 \\
0.500\end{array}$ & $\begin{array}{l}0.960 \\
1.013 \\
3.352^{*} \\
1.013 \\
0.693\end{array}$ & $\begin{array}{r}\mathrm{D} 20-1 \\
2 \\
3 \\
4 \\
5\end{array}$ & $\begin{array}{r}5 \\
2 \\
2 \\
5 \\
17\end{array}$ & $\begin{array}{r}5 \\
4 \\
5 \\
12 \\
10\end{array}$ & $\begin{array}{l}0.500 \\
0.344 \\
0.227 \\
0.072 \\
0.124\end{array}$ & $\begin{array}{l}0.693 \\
1.067 \\
1.483 \\
2.631 \\
2.087\end{array}$ \\
\hline $\begin{array}{r}\mathrm{C} 105-1 \\
2 \\
3 \\
4 \\
5\end{array}$ & $\begin{array}{l}3 \\
2 \\
4 \\
7 \\
1\end{array}$ & $\begin{array}{l}2 \\
3 \\
7 \\
4 \\
2\end{array}$ & $\begin{array}{l}0.500 \\
0.500 \\
0.274 \\
0.274 \\
0.500\end{array}$ & $\begin{array}{l}0.693 \\
0.693 \\
1.295 \\
1.295 \\
0.693\end{array}$ & $\begin{array}{r}\mathrm{D} 21-1 \\
2 \\
3 \\
4 \\
5\end{array}$ & $\begin{array}{r}12 \\
3 \\
3 \\
12 \\
3\end{array}$ & $\begin{array}{r}10 \\
9 \\
2 \\
9 \\
3\end{array}$ & $\begin{array}{l}0.416 \\
0.073 \\
0.500 \\
0.332 \\
0.500\end{array}$ & $\begin{array}{l}0.877 \\
2.617 \\
0.693 \\
1.103 \\
0.693\end{array}$ \\
\hline $\begin{array}{r}\mathrm{C} 106-1 \\
2 \\
3 \\
4 \\
5\end{array}$ & $\begin{array}{r}2 \\
6 \\
9 \\
8 \\
13\end{array}$ & $\begin{array}{r}4 \\
9 \\
8 \\
7 \\
11\end{array}$ & $\begin{array}{l}0.344 \\
0.304 \\
0.500 \\
0.500 \\
0.419\end{array}$ & $\begin{array}{l}1.067 \\
1.191 \\
0.693 \\
0.693 \\
0.870\end{array}$ & $\begin{array}{r}\text { D23-1 } \\
2 \\
3 \\
4 \\
5\end{array}$ & $\begin{array}{l}5 \\
6 \\
1 \\
1 \\
5\end{array}$ & $\begin{array}{l}3 \\
6 \\
0 \\
1 \\
7\end{array}$ & $\begin{array}{l}0.363 \\
0.500 \\
\\
0.500 \\
0.387\end{array}$ & $\begin{array}{l}1.013 \\
0.693 \\
0.693 \\
0.950\end{array}$ \\
\hline $\begin{array}{r}\mathrm{C} 107-1 \\
2 \\
3 \\
4 \\
5\end{array}$ & $\begin{array}{r}11 \\
12 \\
4 \\
12 \\
8\end{array}$ & $\begin{array}{l}6 \\
9 \\
5 \\
6 \\
5\end{array}$ & $\begin{array}{l}0.166 \\
0.332 \\
0.500 \\
0.119 \\
0.291\end{array}$ & $\begin{array}{l}1.796 \\
1.103 \\
0.693 \\
2.129 \\
1.234\end{array}$ & $\begin{array}{r}\mathrm{D} 25-1 \\
2 \\
3 \\
4 \\
5\end{array}$ & $\begin{array}{l}1 \\
3 \\
4 \\
0 \\
1\end{array}$ & $\begin{array}{l}3 \\
0 \\
5 \\
0 \\
2\end{array}$ & $\begin{array}{l}0.313 \\
0.125 \\
0.500 \\
\\
0.500\end{array}$ & $\begin{array}{l}1.167 \\
2.080 \\
0.693 \\
0.693\end{array}$ \\
\hline $\begin{array}{r}\mathrm{C} 123-1 \\
2 \\
3 \\
4 \\
5\end{array}$ & $\begin{array}{r}10 \\
14 \\
15 \\
3 \\
1\end{array}$ & $\begin{array}{r}9 \\
16 \\
9 \\
7 \\
3\end{array}$ & $\begin{array}{l}0.500 \\
0.428 \\
0.154 \\
0.172 \\
0.313\end{array}$ & $\begin{array}{l}0.693 \\
0.849 \\
1.871 \\
1.760 \\
1.161\end{array}$ & $\begin{array}{r}\text { D28-1 } \\
2 \\
3 \\
4 \\
5\end{array}$ & $\begin{array}{l}4 \\
7 \\
4 \\
9 \\
0\end{array}$ & $\begin{array}{r}2 \\
10 \\
4 \\
7 \\
0\end{array}$ & $\begin{array}{l}0.344 \\
0.315 \\
0.500 \\
0.402\end{array}$ & $\begin{array}{l}1.067 \\
1.155 \\
0.693 \\
0.911\end{array}$ \\
\hline $\begin{array}{r}\mathrm{E} 22-1 \\
2 \\
3 \\
4 \\
5\end{array}$ & $\begin{array}{l}4 \\
1 \\
1 \\
1 \\
2\end{array}$ & $\begin{array}{l}2 \\
2 \\
2 \\
1 \\
0\end{array}$ & $\begin{array}{l}0.344 \\
0.500 \\
0.500 \\
0.500 \\
0.250\end{array}$ & $\begin{array}{l}1.067 \\
0.693 \\
0.693 \\
0.693 \\
1.386\end{array}$ & $\begin{array}{r}\text { L } 2-1 \\
2 \\
3 \\
4 \\
4 \\
5\end{array}$ & $\begin{array}{l}2 \\
1 \\
2 \\
4 \\
1\end{array}$ & $\begin{array}{l}0 \\
3 \\
4 \\
6 \\
1\end{array}$ & $\begin{array}{l}0.250 \\
0.313 \\
0.344 \\
0.377 \\
0.500\end{array}$ & $\begin{array}{l}1.386 \\
1.161 \\
1.067 \\
0.975 \\
0.693\end{array}$ \\
\hline $\begin{array}{r}\mathrm{E} 23-1 \\
\end{array}$ & $\begin{array}{l}7 \\
1\end{array}$ & $\begin{array}{l}9 \\
1\end{array}$ & $\begin{array}{l}0.402 \\
0.500\end{array}$ & $\begin{array}{l}0.911 \\
0.693\end{array}$ & $\begin{array}{lr}\text { L } & 5-1 \\
& 2\end{array}$ & $\begin{array}{l}0 \\
4\end{array}$ & $\begin{array}{l}1 \\
1\end{array}$ & 0.188 & 1.671 \\
\hline
\end{tabular}


Appendix Table. (continued)

\begin{tabular}{|c|c|c|c|c|c|c|c|c|c|}
\hline & $b$ & se & p & $-\log _{e}$ & & $b$ & se & $\mathrm{p}$ & $-\log _{e}$ \\
\hline $\begin{array}{l}3 \\
4 \\
5\end{array}$ & $\begin{array}{l}2 \\
1 \\
4\end{array}$ & $\begin{array}{l}2 \\
1 \\
0\end{array}$ & $\begin{array}{l}0.500 \\
0.500 \\
0.063\end{array}$ & $\begin{array}{l}0.693 \\
0.693 \\
2.765\end{array}$ & $\begin{array}{l}3 \\
4 \\
5\end{array}$ & $\begin{array}{l}6 \\
0 \\
2\end{array}$ & $\begin{array}{l}5 \\
1 \\
0\end{array}$ & $\begin{array}{l}0.500 \\
0.250\end{array}$ & $\begin{array}{l}0.693 \\
1.386\end{array}$ \\
\hline \multirow[t]{3}{*}{$\begin{array}{r}\mathrm{E} 24-1 \\
2 \\
3 \\
4 \\
5\end{array}$} & $\begin{array}{l}9 \\
2 \\
4 \\
7 \\
3\end{array}$ & $\begin{array}{l}4 \\
3 \\
3 \\
2 \\
9\end{array}$ & $\begin{array}{l}0.133 \\
0.500 \\
0.500 \\
0.090 \\
0.073\end{array}$ & $\begin{array}{l}2.017 \\
0.693 \\
0.693 \\
2.408 \\
2.617\end{array}$ & $\begin{array}{r}\text { L } 7-1 \\
2 \\
3 \\
4 \\
5\end{array}$ & $\begin{array}{l}0 \\
0 \\
7 \\
2 \\
0\end{array}$ & $\begin{array}{l}0 \\
0 \\
7 \\
1 \\
0\end{array}$ & $\begin{array}{l}0.500 \\
0.500\end{array}$ & $\begin{array}{l}0.693 \\
0.693\end{array}$ \\
\hline & & & & & $\begin{array}{r}\text { L 8-1 } \\
2 \\
3 \\
4 \\
5\end{array}$ & $\begin{array}{r}3 \\
14 \\
16 \\
4 \\
14\end{array}$ & $\begin{array}{r}3 \\
15 \\
10 \\
0 \\
5\end{array}$ & $\begin{array}{l}0.500 \\
0.500 \\
0.164 \\
0.063 \\
0.032\end{array}$ & $\begin{array}{l}0.693 \\
0.693 \\
1.808 \\
2.765 \\
3.442^{*}\end{array}$ \\
\hline & & & & & $\begin{array}{r}\text { L 10-1 } \\
2 \\
3 \\
4 \\
5\end{array}$ & $\begin{array}{l}3 \\
3 \\
0 \\
4 \\
0\end{array}$ & $\begin{array}{l}1 \\
2 \\
0 \\
1 \\
0\end{array}$ & $\begin{array}{l}0.313 \\
0.500 \\
0.188\end{array}$ & $\begin{array}{l}1.161 \\
0.693 \\
1.671\end{array}$ \\
\hline
\end{tabular}

p: Probabilities of obtaining the observed distribution of complementary crossover classes in each family.

The $-\log _{\theta}$ is equal to $\chi^{2} / 2$ associated with 2 degrees of freedom. 